\title{
Traditional Chinese Medicine Prescriptions Decrease Diarrhea Rate by Relieving Colonic Inflammation and Ameliorating Caecum Microbiota in Piglets
}

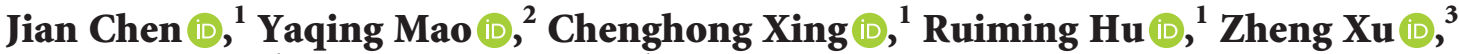 \\ Huabin Cao $\left(\mathbb{1},{ }^{1}\right.$ and Junrong Luo $\mathbb{1}^{1}$ \\ ${ }^{1}$ Jiangxi Provincial Key Laboratory for Animal Health, Institute of Animal Population Health, \\ College of Animal Science and Technology, Jiangxi Agricultural University, Nanchang, Jiangxi, China \\ ${ }^{2}$ China Institute of Veterinary Drug Control (MOA Center for Veterinary Drug Evaluation), Beijing, China \\ ${ }^{3}$ Department of Mathematics and Statistics, Wright State University Dayton, Dayton, OH 45435, USA
}

Correspondence should be addressed to Huabin Cao; chbin20020804@jxau.edu.cn and Junrong Luo; junrluo@mail.jxau.edu.cn

Received 22 October 2019; Revised 9 March 2020; Accepted 24 March 2020; Published 14 April 2020

Academic Editor: Raffaele Pezzani

Copyright (c) 2020 Jian Chen et al. This is an open access article distributed under the Creative Commons Attribution License, which permits unrestricted use, distribution, and reproduction in any medium, provided the original work is properly cited.

Diarrhea is a leading cause of death in piglets. XiaoJianZhong (XJZ) and Jingsananli-sepsis (JSS) were two traditional Chinese medicine (TCM) prescriptions to prevent and treat intestinal diseases, including diarrhea and inflammatory disease. Here, we investigated the effects of XJZ and JSS on diarrhea rate, growth performance, colonic inflammation, and caecum microbiota in piglets. A total of 18 piglets were selected and randomly divided into three groups. Control group was supplied with basal diets, while TCM1 and TCM2 groups were, respectively, supplied with XJZ and JSS in basal diets. Decreased diarrhea rate, colonic or caecal $\mathrm{pH}$, and elevated apparent nutrient digestibility were observed in both TCM groups. Meanwhile, both prescriptions alleviated colonic inflammation by decreasing mRNA expression of proinflammatory cytokines and suppressing the TLR4/ MyD88/NF- $\kappa$ B signaling pathway. Additionally, TCM1 and TCM2 prescriptions ameliorated caecum microbiota composition and increased the abundance of beneficial bacteria, together with regulations on several genes that are responsible for signaling pathways involved in cancers and metabolic diseases. Importantly, both TCM1 and TCM2 significantly promoted the average daily gain $(A D G)$ and reduced the feed: gain $(F: G)$ ratio. In conclusion, both TCM prescriptions effectively decreased diarrhea rate and increased growth performance by elevating apparent nutrient digestibility and gut health, via relieving colonic inflammation and ameliorating gut microbiota composition of piglets.

\section{Introduction}

It is well-known that piglets' nursery is the most critical period in the pig breeding process. However, in piglets, the gut immune system is easily disturbed because of immature immunity, deficiency of digestive ability, and enteric antigenic challenges [1]. When the external environment fluctuates, the intestinal immunity of piglets is prone to disorders leading to diarrhea, which is one of the major causes of body weight loss or even death in nursery piglets [2]. Antibiotics as feed additive have been used to promote daily gain and prevent or treat bacterial diarrhea in recent years [3]. However, the misuse and abuse of antibiotics have led to antibiotic residue and multiantibiotic resistance [4]. Thus, it is necessary to develop alternative methods to promote growth performance or antidiarrhea of piglet.

Traditional Chinese medicine (TCM) is composed of derivatives from natural plants. The pharmaceutical activities and clinical benefits of these natural ingredients have been demonstrated and witnessed for long-time clinical application [5]. Multiple active compounds in TCM could hit various targets and exert synergistic therapeutic efficacies [6]. According to the principles of TCM, typically, formulae consist of several types of medicinal herbs or minerals, in 
which one represents the principal component and the others serve as adjuvants to assist in the effects or facilitate the delivery of the principal component $[7,8]$. Due to the complicated pathogenesis and progression of diarrhea, the therapeutic effect of a single herb may be modest in the clinic [9]. As the classical TCM prescriptions, XiaoJianZhong (XJZ) prescription and Jingsananli-sepsis (JSS) prescription are employed to prevent and treat digestive system diseases for hundreds of years, including diarrhea and enteritidis by promoting the physiological function of the spleen and stomach [8]. However, the mechanism of previously determined TCM prescriptions on ameliorating intestine health and physiology in piglets has not been clarified.

Recent study has shown that inflammatory damages toward the intestinal barrier were the main intrinsic reason of diarrhea in piglets [1]. Intestinal inflammatory response is mainly mediated by the TLR4/MyD88/NF- $\kappa \mathrm{B}$ signaling pathway, which enhances the production of various proinflammatory cytokines (i.e., IL- $1 \beta$, IL- 6 , and TNF- $\alpha$ ) [10]. On the other hand, increasing evidence highlights the cardinal role of gut microbiota in inflammatory response and immunotherapy $[11,12]$, due to their intrinsic capacity of drug metabolism and the influence on host metabolizing homeostasis [13]. As reported, dietary amino acids (AAs), as an ingredient in TCM, are considered as the medium for interaction between host and microorganism [14]. Meanwhile, gut microbiota have been considered as a forgotten organ for the disposition of herbal ingredients. Liu et al. [11] demonstrated a traditional Chinese herbal formula of Shen Ling Bai Zhu (some components are same as this experiment, e.g., Poria, Glycyrrhiza, and Atractylodes) increased the number of beneficial bacteria, such as Bifidobacterium and Lactobacillus, which may act directly or indirectly on the course of diarrhea in the gut [11]. Thus, we speculated that regulating gut microbiota composition and alleviating intestinal inflammation by TCM prescription may be a new strategy for antidiarrhea in piglets. However, the effects of all previously determined TCM prescriptions on intestinal inflammation and gut microbiota in piglets have not been clarified.

Thus, the objectives of this study were to evaluate the effects of both TCM prescriptions as feed additives on growth performance and diarrhea in piglets, and to determine whether TCM prescriptions could ameliorate health status of the large intestine by alleviating colonic inflammation response and regulating caecal microbiota.

\section{Materials and Methods}

2.1. Animals, Experimental Design, and Ratios. This trial was carried out in the Researching and Teaching Base of Jiangxi Agricultural University for 60 days, and the experimental design was a complete randomized design. Consistent with Shu et al. [15], diarrhea index was scored according to the fecal: 0 , normal; 1 , nonformed pellets; 2 , soft feces; 3 , semisolid containing more than half waterlike feces; and 4, water-like feces. The diarrhea rate was calculated every day based on diarrhea index (diarrhea index $\geq 3)$. A total of 18 piglets $(17.37 \pm 1.32 \mathrm{~kg}$, large white $\times$ landrace $\times$ Duroc) at 40 days of age (weaned at 5 weeks of age and 5 days for adaptation) were randomly allocated into three groups based on body weight and gender $(n=6)$. Experimental group included (a) basal diet (Control), (b) basal diet supplemented with $10 \mathrm{~g} / \mathrm{kg} \mathrm{XJZ}$ prescription (TCM1), or (c) basal diet supplemented with $3 \mathrm{~g} / \mathrm{kg}$ JSS prescription (TCM2), respectively. The dose of TCM1 $(10 \mathrm{~g} / \mathrm{kg})$ and TCM2 $(3 \mathrm{~g} / \mathrm{kg})$ was assessed based on our preliminary tests and traditional Chinese pharmacopoeia (2005). Basal diets were formulated to meet the nutrient requirements of piglets (NRC, 2012). All the raw materials for TCM1 and TCM2 were provided by The Spirit Jinyu Biological Pharmaceutical Co., Ltd (Huhhot, Inner Mongolia, China). All dried Chinese herbs were smashed through a $2.5 \mathrm{~mm}$ screen sieve. Composition and main active constituents of TCM1 and TCM2 are presented in Table 1.

2.2. Sample Collection and Gut pH Value Detection. Fresh fecal samples (200-300 g per sample) were collected on the ground during the last 3 days of the experimental period. On the last day of the experiment, all piglets were killed by euthanasia with an intravenous injection of sodium pentobarbital $(40 \mathrm{mg} / \mathrm{kg} \cdot \mathrm{BW})$. Then, the abdominal cavity was opened for sample collection. Segments of midcolon tissue and midcaecum contents were harvested immediately after euthanasia, rapidly frozen in liquid nitrogen, and stored at $-80^{\circ} \mathrm{C}$ for various analyses. Meanwhile, use a mobile $\mathrm{pH}$ detector (pH-STAR) to measure $\mathrm{pH}$ values of the midcolon and midcaecum contents. All experimental protocols were approved by the Committee for the Care and Use of Experimental Animals, Jiangxi Agricultural University, Jiangxi, China.

2.3. Measurement of Apparent Nutrient Digestibility. Feed and fecal samples were dried to constant weight at $65^{\circ} \mathrm{C}$ for 72 hours in a forced air oven. The treatment of feces and feed samples and the measurement of crude protein (CP), crude fat (CF), neutral detergent fiber (NDF), acid detergent fiber $(\mathrm{ADF})$, and $\mathrm{Ca}$ and $\mathrm{P}$ apparent digestibility were consistent with the description in Guo et al. [16].

2.4. Determination of the Growth Performance. Daily feed intakes were recorded, and body weights of piglets on an empty stomach were measured on days 1 and 60 of the experimental period. On the basis of these data, average daily gain (ADG), average daily feed intake (ADFI), and the feed: gain $(F: G)$ ratio were calculated.

2.5. Quantitative Real-Time PCR. The mRNA expression of inflammation-related genes (TLR4, MyD88, NF- $\kappa$ B, IL-6, IL-8, IL-10, and TNF- $\alpha$ ) in the TLR/MyD88/NF- $\kappa$ B signaling pathway in the colon was determined by quantitative real-time PCR (qRT-PCR). The total RNA was isolated using the TransZol Up Reagent (TransGen Biotech, Beijing, China). In brief, cDNA was synthesized using a TransScript ${ }^{\circledR}$ One-Step gDNA Removal and cDNA Synthesis SuperMix 
TABLE 1: Composition and main active constituents of TCM1 and TCM2 (air-dried basis) ${ }^{\dagger}$.

\begin{tabular}{|c|c|c|c|}
\hline Latin name & Main active constituent & Used part & Content (\%) \\
\hline \multicolumn{4}{|c|}{ TCM1 } \\
\hline Cassia twig & Cinnamaldehyde & Dried twig & 13 \\
\hline Glycyrrhiza uralensis & Glycyrrhizin & Dried root & 4 \\
\hline Ziziphus zizyphus & Jujuba polysaccharide & Dried fructification & 4 \\
\hline Cynanchum otophyllum & Paeoniflorin & Dried root & 13 \\
\hline Zingiber officinale Roscoe & Ginger oleoresin & Dried root & 6 \\
\hline Rhizoma Atractylodes & Atractylodine & Dried root & 14 \\
\hline Atractylodes macrocephala & Biatractylolide & Dried root & 10.5 \\
\hline Poria cocos & Pachymaran & Dried sclerotium & 10.5 \\
\hline Coptis chinensis Franch. & Berberine & Dried root & 4 \\
\hline Maltose & Maltose & - & 21 \\
\hline Total & & & 100 \\
\hline \multicolumn{4}{|c|}{ TCM2 } \\
\hline Nepeta cataria L. & Nepeta cataria oil & Dried stem & 16.5 \\
\hline Radix Saposhnikoviae & Chromone glycoside & Dried root & 16.5 \\
\hline Notopterygium incisum & Notopterol & Dried root & 16.5 \\
\hline Radix Angelicae pubescentis & Heraclenin & Dried root & 16.5 \\
\hline Radix bupleuri & Saikosaponin & Dried root & 10 \\
\hline Radix Peucedani & Peucedanin & Dried root & 10 \\
\hline Poria cocos & Pachymaran & Dried sclerotium & 10 \\
\hline Glycyrrhiza uralensis & Glycyrrhizin & Dried root & 4 \\
\hline Total & & & 100 \\
\hline
\end{tabular}

Note. ${ }^{\dagger}$ Main active constituents of TCM come from Chinese pharmacopoeia (2005).

reagent kit (TransGen Biotech, Beijing, China) according to the kit's instructions. Then, it was stored at $-20^{\circ} \mathrm{C}$ for SYBR Green qRT-PCR. Gene-specific primers of all genes were designed using the Primer Premier software (PREMIER Biosoft International, CA, USA). The housekeeping gene GAPDH was used as an internal reference, and the primer sequences are shown in Table 2. The RT-qPCR profiles were as follows: $95^{\circ} \mathrm{C}$ for 10 minutes, 40 cycles at $95^{\circ} \mathrm{C}$ for 15 seconds, $60^{\circ} \mathrm{C}$ for 60 seconds, and extension at $95^{\circ} \mathrm{C}$ for 15 seconds.

2.6. Western Blot. Total protein was extracted with RIPA Lysis buffer (Applygen, Beijing, China). Protein supernatant was separated by $10 \%$ SDS-PAGE and transferred onto a PVDF membranes. After being blocked with 5\% skimmed milk powder, the membrane was incubated with the appropriate primary antibodies overnight at $4^{\circ} \mathrm{C}$, followed by incubation with the corresponding secondary antibodies for 1 hour at room temperature. The membranes were washed three times for 10 minutes each, incubated with SuperSignal chemiluminescent substrate (Pierce), and imaged by ChemiDoc XRS + Imaging System (Bio-Rad). Blots were semiquantified using BandScan software. Primary antibodies for NF- $\kappa$ B p65 (Cell Signaling, 6956, 1:1000), phospho-NF- $\kappa$ B p65 (Cell Signaling, 3033, $1: 1000)$, I $\kappa \mathrm{B}-\alpha$ (Cell Signaling, 4814, $1: 1000$ ), and phospho-I $\kappa \mathrm{B}-\alpha$ (Cell Signaling, 9246, $1: 1000)$ were used in this study.

2.7. 16S rRNA MiSeq Sequencing of Gut Microbiota. Sequencing service was provided by Personal Biotechnology Co., Ltd., Shanghai, China. Total DNA was isolated from the caecum content samples as previously reported with some modifications [17]. The V3-V4 region of bacterial 16S rRNA gene was amplified by PCR, and the products were separated by gel electrophoresis and purified using the AP-GX-500 DNA Gel Extraction Kit (Axygen, Corning, USA). Library was build up with the obtained products and then sequenced on a MiSeq sequencing platform (Illumina, USA) as described by Zhao et al. [18].

2.8. Bioinformatics Analysis. The trimmed and assembled sequences from each sample were aligned to the Greengene $16 \mathrm{~S}$ rRNA database using the best hit classification option to classify the taxonomy abundance in QIIME [19]. Bacterial operation taxonomic units (OTUs) were generated using the uclust function in QIIME. A Venn diagram was generated to compare OTUs between groups. The following statistics were performed by $\mathrm{R}$ software. ACE, Chao, Simpson, and Shannon indices were calculated for $\alpha$-diversity evaluation. The abundance and diversity of the OTUs ( $\beta$-diversity) were examined using partial least squares discriminant analysis (PLS-DA) and nonmetric multidimensional scaling (NMDS) in $\mathrm{R}$ software. The statistical significance of the separation among groups was assessed by the linear discriminant analysis effect size (LEfSe) method based on linear discriminant analysis (LDA) scores exploited by Curtis Huttenhower (http:// huttenhower.sph.harvard.edu/galaxy/), which used the nonparametric factorial Kruskal-Wallis and Wilcoxon rank sum test to identify key OTUs for separating different treatment groups at a significance level of 0.05 . Microbial functions were predicted using PICRUSt, as described by Jiyan et al. [20]. 
TABle 2: Primers used in this study.

\begin{tabular}{|c|c|c|}
\hline Target & GenBank number & Primers sequence $\left(5^{\prime}-3^{\prime}\right)$ \\
\hline GAPDH & NM_001206359 & $\begin{array}{l}\text { F: ACTCACTCTTCCACTTTTGATGCT } \\
\text { R: TGTTGCTGTAGCCAAATTCA }\end{array}$ \\
\hline TLR4 & NM_001113039.1 & $\begin{array}{c}\text { F: GCCATCGCTGCTAACATCATC } \\
\text { R: CTCATACTCAAAGATACACCATCGG }\end{array}$ \\
\hline MyD88 & NM_001099923.1 & $\begin{array}{c}\text { F: TGGTAGTGGTTGTCTCTGATGA } \\
\text { R: TGGAGAGAGGCTGAGTGCAA }\end{array}$ \\
\hline $\mathrm{NF}-\kappa \mathrm{B}$ & NM_001048232.1 & $\begin{array}{l}\text { F: CTCGCACAAGGAGACATGAA } \\
\text { R: ACTCAGCCGGAAGGCATTAT }\end{array}$ \\
\hline IL-6 & NM_001252429.1 & $\begin{array}{l}\text { F: TGGCTACTGCCTTCCCTACC } \\
\text { R: CAGAGATTTTGCCGAGGATG }\end{array}$ \\
\hline IL-8 & NM_213867.1 & $\begin{array}{l}\text { F: TTCGATGCCAGTGCATAAATA } \\
\text { R: CTGTACAACCTTCTGCACCCA }\end{array}$ \\
\hline IL-10 & HQ026020.1 & $\begin{array}{l}\text { F: GCTGAAGACCCTCAGGCTGA } \\
\text { R: GCTGAAGACCCTCAGGCTGA }\end{array}$ \\
\hline TNF- $\alpha$ & NM_214022.1 & $\begin{array}{l}\text { F: CCAATGGGCAGAHTGGGTATG } \\
\text { R: TGAAGAGGACCTGGGAGTAG }\end{array}$ \\
\hline
\end{tabular}

Note. GAPDH, glyceraldehyde-3-phosphate dehydrogenase; TLR4, toll-like receptors 4; MyD88, myeloid differentiation factor 88 ; NF- $\kappa$ B, nuclear factor- $\kappa$ B; IL-6, interleukin-6; IL-8, interleukin-8; IL-10, interleukin-10; TNF- $\alpha$, tumor necrosis factor- $\alpha$.

2.9. Statistical Analysis. Statistical analysis was performed using SPSS 17.0 software (SPSS Inc., Chicago, IL, USA). All results are expressed in the format of mean \pm standard deviation (SD). Comparisons between two or multiple groups were made with Student's $t$-test or ANOVA. $P<0.05$ was considered to be significant, and $P<0.01$ was considered to be highly significant.

\section{Results}

3.1. Diarrhea Index. Diarrhea is the main cause of the decline in the survival rate of nursery piglets. To determine whether TCM could effectively prevent piglets' diarrhea, we calculated the diarrhea rate (Figure 1). After 60 days of treatment, both TCM groups significantly reduced the diarrhea rate in piglets. TCM1 and TCM2 showed a significant reduction of the diarrhea rate compared to the control $(P<0.01)$. However, there was no significant difference between TCM1 and TCM2 treatment $(P>0.05)$.

3.2. Intestinal $p H$ Value. More acidic intestinal environment could inhibit the proliferation of various pathogens. To determine whether TCM could ameliorate the environment of large intestine, we measured the colon and caecum $\mathrm{pH}$ value. Compared to the control, colon and caecum $\mathrm{pH}$ were decreased in different levels after TCM intervention (Table 3). Moreover, TCM1 reduced the colon and caecum $\mathrm{pH}$ by $7.85 \%(P<0.05)$ and $11.54 \%(P<0.01)$, but no difference was noticed on the colon $\mathrm{pH}$ of the TCM2 group $(P>0.05)$ compared to the control. Similarly, the caecum $\mathrm{pH}$ also declined in the TCM2 group extremely significantly $(P<0.01)$.

3.3. Nutrient Apparent Digestibility. To evaluate whether both TCM treatments could improve the apparent digestibility in piglets, we assessed the digestibility of $\mathrm{CP}, \mathrm{CF}, \mathrm{NDF}$, $\mathrm{ADF}, \mathrm{Ca}$, and $\mathrm{P}$ (Table 4). Compared to the control, TCM1

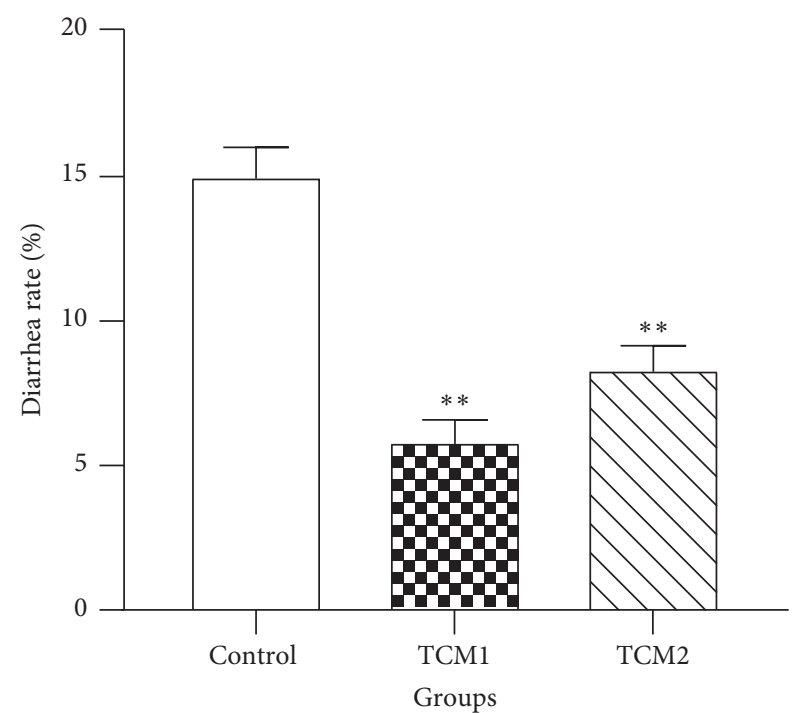

FIGURE 1: Effects of TCM supplements on diarrhea rate of piglets. $* * P<0.01$ vs. the control. The results are expressed as mean $\pm \mathrm{SD}$, one-way ANOVA, $n=6$.

notably improved the apparent nutrient digestibility of $\mathrm{CP}$, $\mathrm{CF}, \mathrm{NDF}, \mathrm{ADF}, \mathrm{Ca}$, and P by $5.04 \%, 6.63 \%, 12.04 \%, 17.12 \%$, 6.56\% $(P<0.01)$, and $6.31 \%(P<0.05)$, respectively. Similarly, the results of $\mathrm{CP}, \mathrm{NDF}$, and $\mathrm{ADF}$ were elevated by $2.56 \%, 7.10 \%(P<0.05)$, and $13.64 \%(P<0.01)$ in the TCM2 group, but no significant difference was observed in $\mathrm{CF}$, $\mathrm{ADF}, \mathrm{Ca}$, and $\mathrm{P}$ levels $(P>0.05)$.

3.4. Growth Performance. To evaluate the effects of TCM1 and TCM2 on growth performance in piglets, we measured the ADG, ADFI, and F: G ratio (Table 5). Compared to the control, ADG was significantly increased in both treatment groups $(P<0.05)$, whereas no difference of ADFI was observed among all groups. Meanwhile, both TCM1 and TCM2 significant decreased the $\mathrm{F}: \mathrm{G}$ ratios $(P<0.01)$. 
TABLE 3: Effects of TCM supplements on intestinal $\mathrm{pH}$ in piglets.

\begin{tabular}{lccc}
\hline Items & \multicolumn{3}{c}{ Groups } \\
& Control & TCM1 & TCM2 \\
\hline Colon pH & $6.62 \pm 0.09^{\mathrm{a}}$ & $6.10 \pm 0.14^{\mathrm{b}}$ & $6.50 \pm 0.21^{\mathrm{ab}}$ \\
Caecum pH & $6.76 \pm 0.07^{\mathrm{A}}$ & $5.98 \pm 0.10^{\mathrm{B}}$ & $6.17 \pm 0.06^{\mathrm{B}}$ \\
\hline
\end{tabular}

Note. Results are expressed as mean $\pm \mathrm{SD}$, one-way ANOVA, $n=6$. ${ }^{\mathrm{a}, \mathrm{b}}$ Within a row, means without a common lowercase superscript are different at $P<0.05$. ${ }^{\mathrm{A}, \mathrm{B}}$ Within a row, means without a common uppercase superscript are different at $P<0.01$.

TABLE 4: Effects of TCM supplements on nutrient digestibility in piglets.

\begin{tabular}{lccc}
\hline Items & Control & Groups \\
& $7 C M 1$ & TCM2 \\
\hline CP (\%) & $74.55 \pm 0.34^{\mathrm{Aa}}$ & $78.51 \pm 0.61^{\mathrm{Bc}}$ & $76.51 \pm 0.30^{\mathrm{Ab}}$ \\
CF (\%) & $73.50 \pm 0.67^{\mathrm{A}}$ & $78.72 \pm 0.58^{\mathrm{B}}$ & $74.57 \pm 0.66^{\mathrm{A}}$ \\
NDF (\%) & $53.39 \pm 1.03^{\mathrm{Aa}}$ & $60.70 \pm 1.22^{\mathrm{Bb}}$ & $57.47 \pm 0.99^{\mathrm{ABb}}$ \\
ADF (\%) & $25.90 \pm 0.67^{\mathrm{A}}$ & $31.25 \pm 0.62^{\mathrm{B}}$ & $29.99 \pm 0.97^{\mathrm{B}}$ \\
Ca (\%) & $58.42 \pm 0.73^{\mathrm{A}}$ & $62.52 \pm 0.73^{\mathrm{B}}$ & $60.50 \pm 0.89^{\mathrm{AB}}$ \\
$\mathrm{P}(\%)$ & $48.83 \pm 1.00^{\mathrm{a}}$ & $52.12 \pm 0.75^{\mathrm{b}}$ & $49.26 \pm 1.01^{\mathrm{a}}$ \\
\hline
\end{tabular}

Note. CP, crude protein; CF, crude fiber; NDF, neutral detergent fiber; ADF, acid detergent fiber. Results are expressed as mean $\pm \mathrm{SD}$, one-way ANOVA, $n=6 .{ }^{\mathrm{a}, \mathrm{b}}$ Within a row, means without a common lowercase superscript are different at $P<0.05$. ${ }^{\mathrm{A}, \mathrm{B}} \mathrm{W}$ ithin a row, means without a common uppercase superscript are different at $P<0.01$.

3.5. mRNA Expression of TLR4/MyD88/NF- $\kappa B$ Signaling Pathway. To determine whether the colonic inflammatory response was alleviated after the TCM intervention, we analysed the mRNA levels of inflammation-related genes in the TLR4/MyD88/NF- $\kappa \mathrm{B}$ signaling pathway. As shown in Figure 2, compared to the control, the TLR4 mRNA level was only downregulated significantly in the TCM1 group $(P<0.05)$, but there was no significant difference in the TCM2 group $(P>0.05)$. Notably, the mRNA expression of MyD88 was decreased significantly in the TCM1 group $(P<0.05)$ and TCM2 group $(P<0.01)$. Additionally, NF- $\kappa \mathrm{B}$ levels of both TCM groups were decreased, but only the TCM2 group showed statistical significance to the control group $(P<0.05)$.

3.6. mRNA Expression of Inflammatory Cytokine Genes. To evaluate the levels of inflammatory cytokines downstream of the TLR4/MyD88/NF- $\kappa$ B signaling pathway, the mRNA expression of IL-6, IL-8, IL-10, and TNF- $\alpha$ was measured (Figure 3). Compared to the control, the expression of IL-6, IL-8, and TNF- $\alpha$ was decreased, whereas IL-10 levels were increased significantly in both TCM prescriptions $(P<0.05$ or $P<0.01)$. Moreover, IL- 8 and TNF- $\alpha$ levels were decreased, and IL-10 levels increased significantly $(P<0.05)$ in the TCM1 group, compared to the TCM2 group.

3.7. Protein Levels of NF- $\kappa B$ Signaling Pathway in the Colon. To further determine the inhibition of NF- $\kappa \mathrm{B}$ signaling pathway in the colon after treating with TCM supplements,
TABLE 5: Effects of TCM treatments on growth performance in piglets.

\begin{tabular}{lccc}
\hline Items & \multicolumn{3}{c}{ Groups } \\
& Control & TCM1 & TCM2 \\
\hline ADG $(\mathrm{kg})$ & $0.52 \pm 0.01^{\mathrm{a}}$ & $0.56 \pm 0.03^{\mathrm{b}}$ & $0.56 \pm 0.02^{\mathrm{b}}$ \\
ADFI (kg) & $0.95 \pm 0.03$ & $0.98 \pm 0.07$ & $1.00 \pm 0.05$ \\
F: G $(\mathrm{kg} / \mathrm{kg})$ & $1.85 \pm 0.06^{\mathrm{A}}$ & $1.75 \pm 0.05^{\mathrm{B}}$ & $1.79 \pm 0.03^{\mathrm{AB}}$ \\
\hline
\end{tabular}

Note. ADG, average daily gain; ADFI, average daily feed intake; F : G: feed: gain. Results are expressed as mean $\pm \mathrm{SD}$, one-way ANOVA, $n=6$.

${ }^{\mathrm{a}, \mathrm{b}}$ Within a row, means without a common lowercase superscript are different at $P<0.05$. ${ }^{\mathrm{A}, \mathrm{B}}$ Within a row, means without a common uppercase superscript are different at $P<0.01$.

the protein levels of NF- $\kappa \mathrm{B}$ p $65, \mathrm{p}-\mathrm{NF}-\kappa \mathrm{B}$ p $65, \mathrm{I} \kappa \mathrm{B}-\alpha$, and $\mathrm{p}-\mathrm{I} \kappa \mathrm{B}-\alpha$ were analysed. Compared to the control, both TCM1 and TCM2 that were highly significant decreased the protein levels of NF- $\kappa \mathrm{B}$ p $65, \mathrm{p}-\mathrm{NF}-\kappa \mathrm{B}$ p65, and $\mathrm{p}-\mathrm{I} \kappa \mathrm{B}-\alpha$ $(P<0.05$ or $P<0.01)$, respectively (Figure 4$)$, while that of I $\kappa \mathrm{B}-\alpha$ protein level was remarkably elevated in the TCM1 $(P<0.01)$ and TCM2 $(P<0.05)$ groups. Importantly, both TCM1 and TCM2 reduced the ratio of phosphorylated protein (p-NF- $\kappa \mathrm{B}$ and $\mathrm{p}-\mathrm{I} \kappa \mathrm{B}-\alpha)$ to total protein significantly $(P<0.01)$. Meanwhile, the ratio of $\mathrm{p}-\mathrm{NF}-\kappa \mathrm{B} / \mathrm{NF}-\kappa \mathrm{B}$ and $\mathrm{p}-\mathrm{I} \kappa \mathrm{B}-\alpha / \mathrm{I} \kappa \mathrm{B}-\alpha$ of the TCM2 group was remarkably higher than the TCM1 group.

3.8. Caecum Microbiota DNA Sequence Data. To evaluate the effect of TCM1 and TCM2 prescriptions on the caecum microflora, we performed 16s RNA sequencing on the caecum contents of piglets. After quality control on the original sequence, 763938 valid sequences in total (on average 42441 sequences per sample) were obtained. Rarefaction analysis results showed that this sequencing depth was sufficient to study the microbial diversity of each sample (Figure 5(a)). Common OTU analysis presented by Venn diagram indicated that there were 404 unique OTUs in the control, 285 in the TCM1 group, and 420 in the TCM2 group, respectively, while 2130 common OTUs were identified in all samples (Figure 5(b)).

3.9. $\alpha$-Diversity and $\beta$-Diversity of the Caecum Microbiota. As shown in Figure 5(c), the Shannon and Simpson indexes of TCM1 showed an higher species diversity than control, but the difference was not significant $(P>0.05)$. The ACE and Chaol indexes of both TCM1 and TCM2 were significantly higher than the control group $(P<0.05)$. Meanwhile, TCM2 elevated $(P<0.05)$ the Simpson index significantly compared to the control.

$\beta$-Analysis was used to compare the similarity of overall community structure, which employed several unsupervised multivariate statistical assessments, including UniFrac NMDS and PLS-DA. The $\beta$-diversity maps showed that the similarity in species diversity was remarkably different between the both TCM groups and the control group. As shown in Figures 5(d) and 5(e), there was an apparent distance of piglet caecum flora among the TCM1, TCM2, and control group. In addition, the group spacing in the 


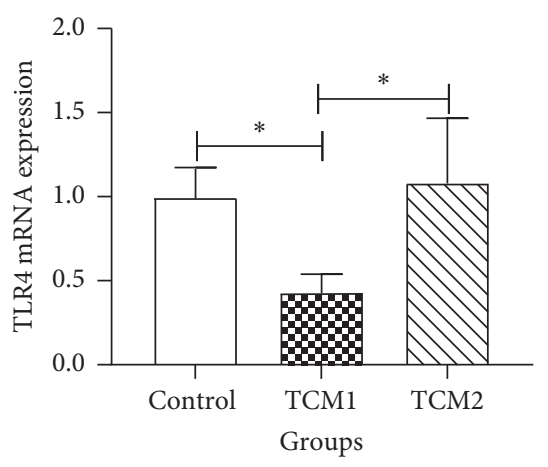

(a)

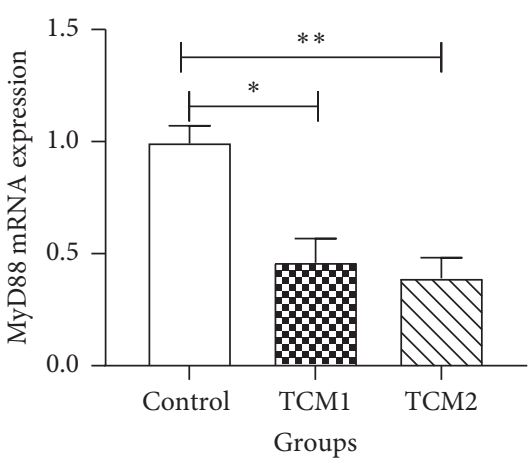

(b)

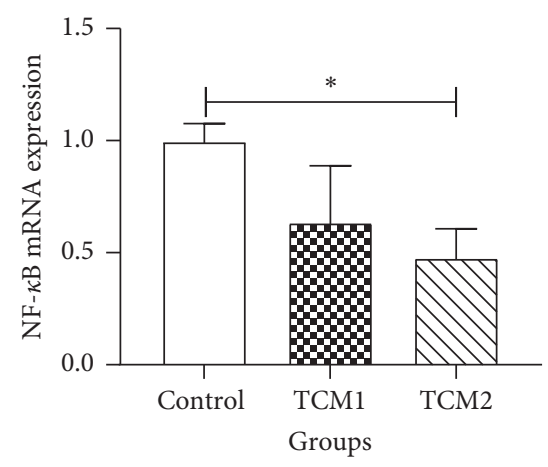

(c)

Figure 2: Effects of both TCM supplements on mRNA expression of (a) TLR4, (b) MyD88, and (c) NF- $\kappa$ B in the colon of piglets. “*” and “**” indicate a significant difference $(P<0.05)$ or a highly significant difference $(P<0.01)$ between treatment groups, respectively. The results are expressed as mean $\pm \mathrm{SD}$, one-way ANOVA, $n=6$.

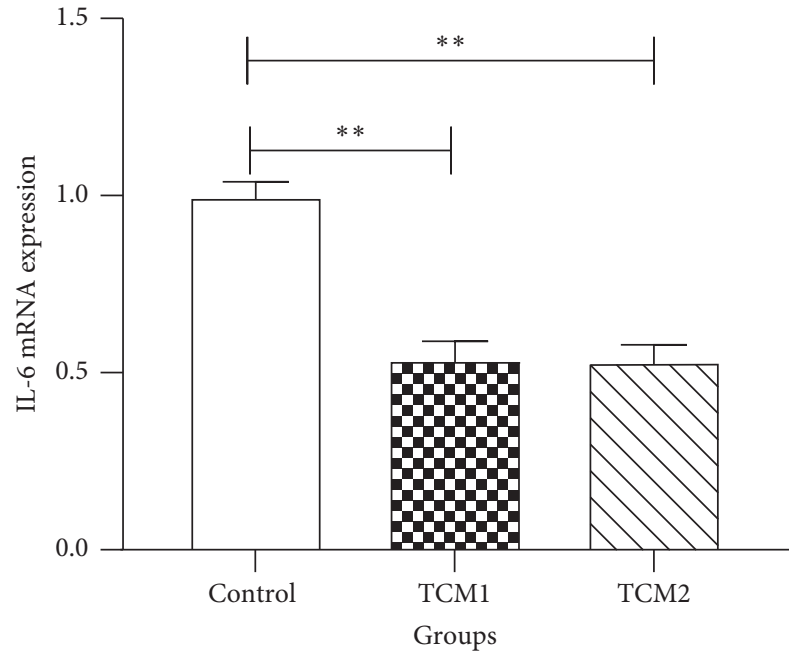

(a)

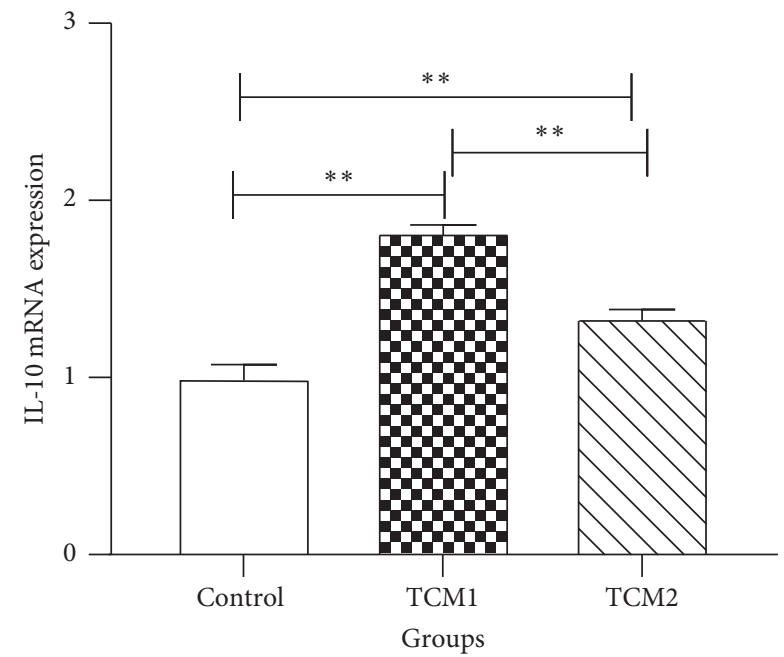

(c)

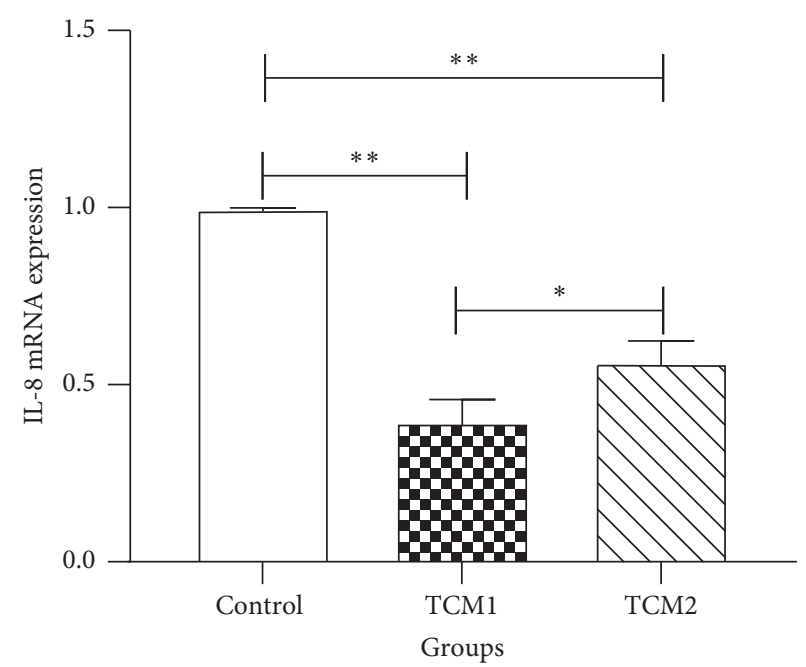

(b)

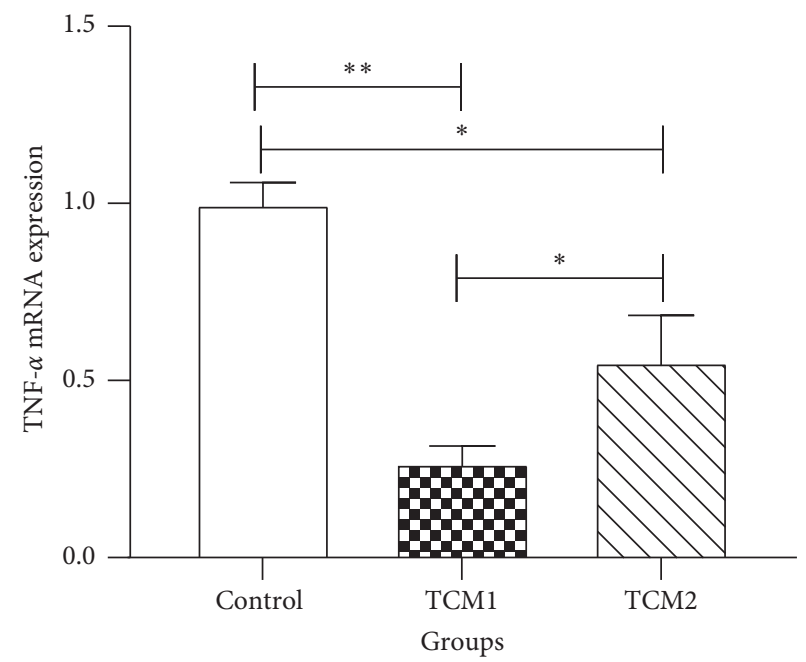

(d)

FIGURE 3: Effects of both TCM supplements on mRNA expression of inflammatory cytokines in the colon of piglets: (a) IL-6; (b) IL-8; (c) IL10; (d) TNF- $\alpha$. “*” and “**” indicate a significant difference $(P<0.05)$ or a highly significant difference $(P<0.01)$ between treatment groups, respectively. The results are expressed as mean $\pm \mathrm{SD}$, one-way ANOVA, $n=6$. 

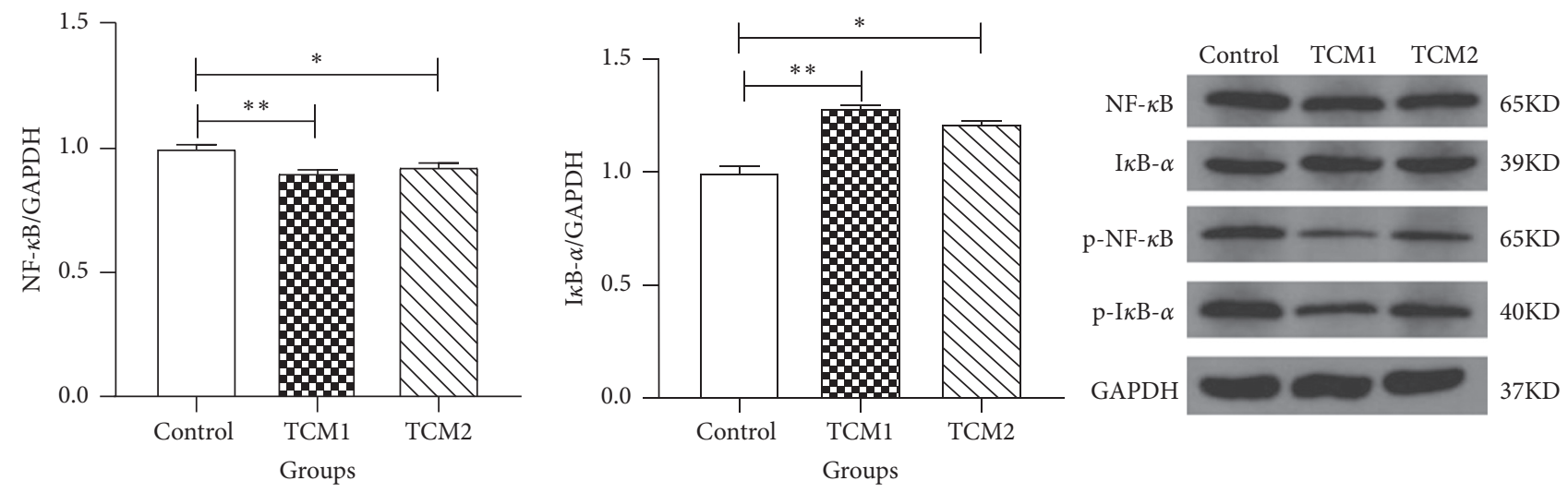

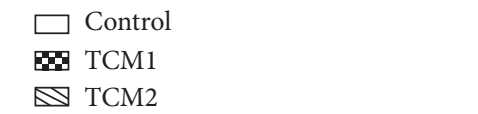

(a)

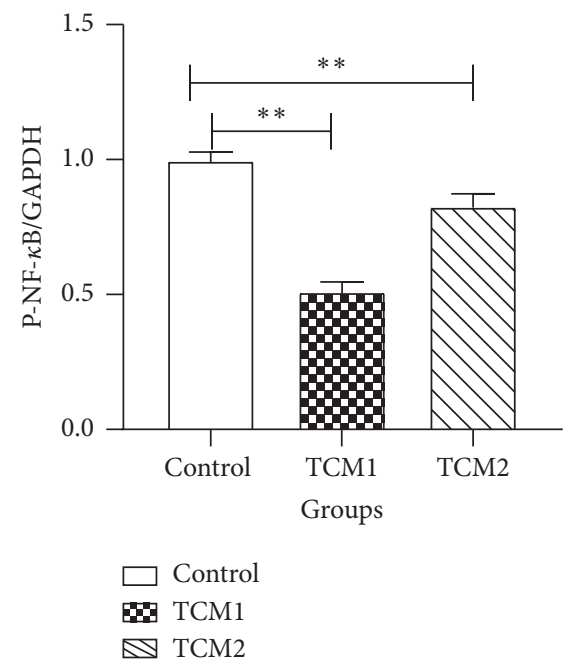

(c)

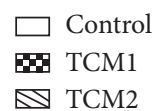

$\$$ TCM2

(b)

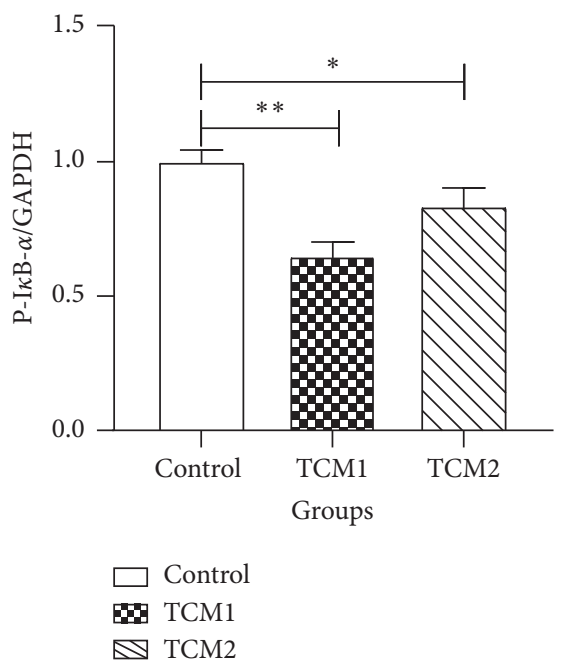

(d)

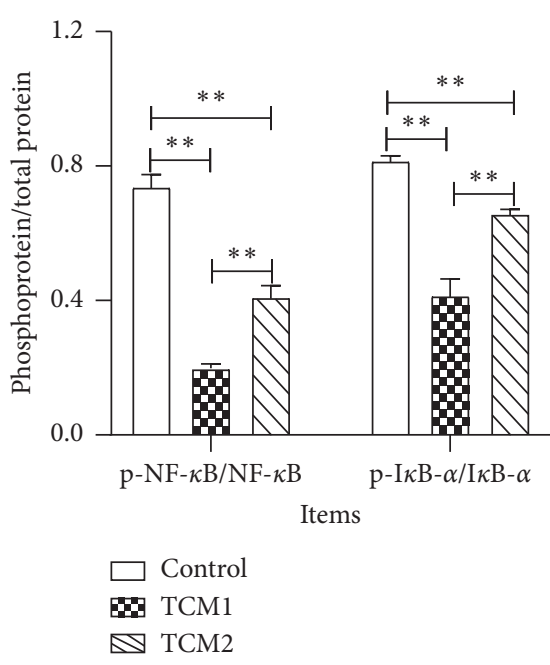

(e)

FIGURE 4: Effect of both TCM supplements on protein expression of NF- $\kappa$ B signaling pathway in colon of piglets: (a) NF- $\kappa \mathrm{B} / \mathrm{GAPDH}$; (b) $\mathrm{I} \kappa \mathrm{B}-\alpha / \mathrm{GAPDH}$; (c) p-NF- $\kappa \mathrm{B} / \mathrm{GAPDH}$; (d) p-I $\kappa \mathrm{B}-\alpha / \mathrm{GAPDH}$; (e) phosphoprotein/total protein. “ $*$ " and “**” indicate a significant difference $(P<0.05)$ or a highly significant difference $(P<0.01)$ between treatment groups, respectively; the results are expressed as mean \pm SD, one-way ANOVA, $n=6$.

TCM treatment groups was smaller than that between the treatment groups and the control group.

3.10. Key Community of Caecum Microbiota. LDA score (Figure 6(a)) higher than 2 indicated a higher relative abundance in the corresponding group than in the other two groups $(P<0.05)$. The relative abundance of significantly different species $(P<0.05)$ was showed by LEfSe taxonomy cladogram (Figure 6(b)). The significantly different communities $(P<0.05)$ belonged to Cyanobacteria and Actinobacteria when supplementation of TCM1. At the genus level, TCM1 showed significant $(P<0.05)$ selective enrichment of Eubacterium, Bulleidia, and Catenibacterium, with the LAD scores of $3.31,3.16$, and 2.88, respectively, and
TCM2 demonstrated a significant $(P<0.05)$ effect on Streptococcus and Bifidobacterium, with the LAD scores of 4.69 and 3.71, respectively.

3.11. Microbiome Function Regulation by TCM Treatment. Comparing the sequencing data with KEGG pathway database by PICRUSt (Figure 7), it was found that both TCM groups significant upregulated $(P<0.05)$ the abundances of genes responsible for transcription (Genetic Information Processing), but downregulated $(P<0.05$ or $P<0.01)$ genes involved in the "cancers" and "metabolic diseases" pathways. Meanwhile, TCM2 also significantly upregulated $(P<0.01)$ abundant genes responsible for lipid metabolism. 


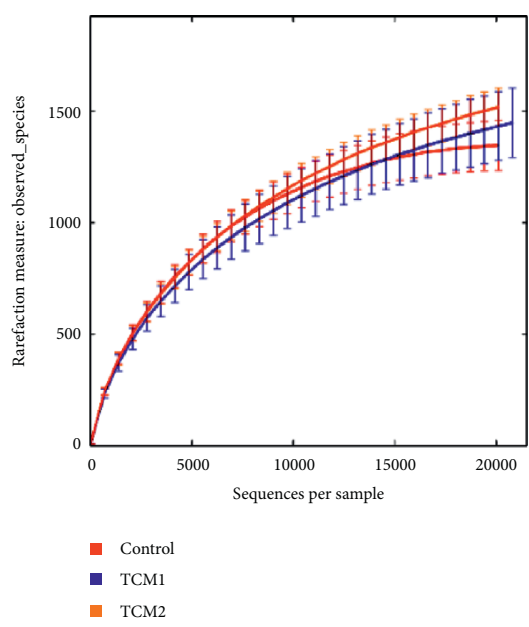

(a)

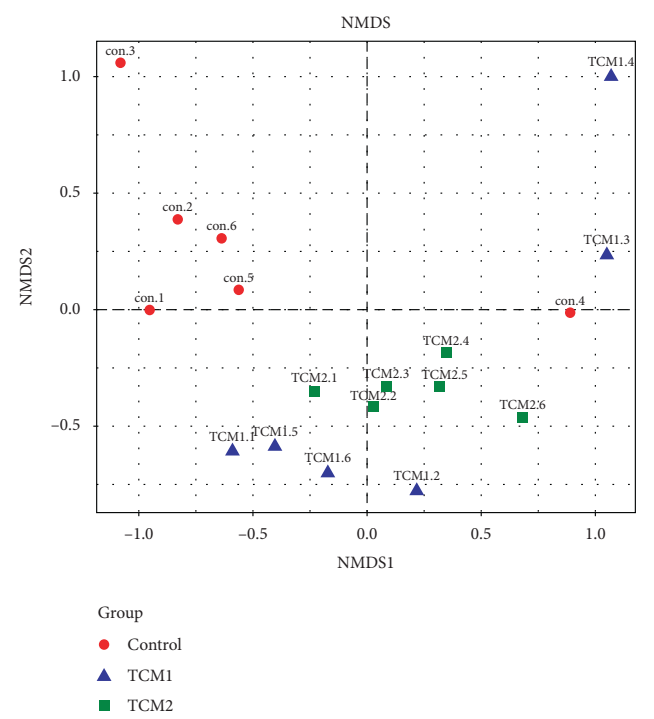

(d)

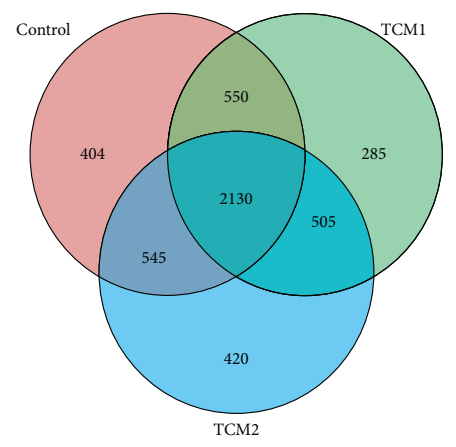

(b)
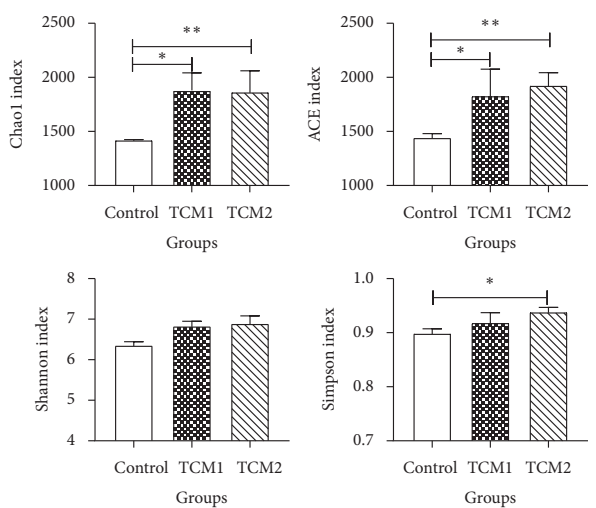

(c)

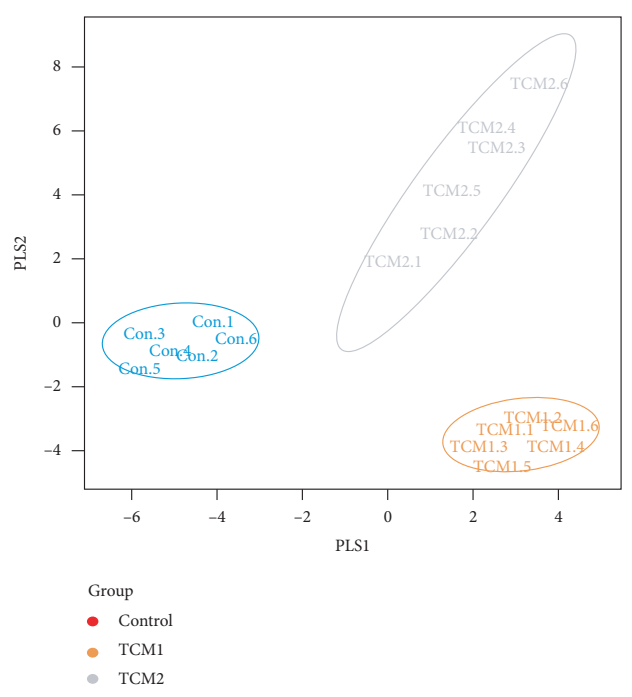

(e)

FiguRE 5: $\alpha$-Diversity and $\beta$-diversity analysis on the caecum microbiota. (a) Rarefaction curves; red, blue, and orange indicate the 6 samples of control, TCM1, and TCM2 groups, respectively. (b) Venn diagram; each circle represents a set of samples; the group between the circle and circle overlapping part digital represents of the common OTUs, and there is no overlapping part representing unique OTUs in each group. (c) Alpha diversity indices comparison; the ACE and Chaol indexes represent the community richness of the microbiota, and the Shannon and Simpson indexes represent the community diversity of the microbiota. Results are expressed as mean \pm SD, one-way ANOVA, $n=6 .{ }^{*} P<0.05$ vs. the control; ${ }^{* *} P<0.01$ vs. the control. (d) UniFrac distance-based nonmetric multidimensional scaling (NMDS); red circle, the control group; blue triangle, the TCM1 group; green square, the TCM2 group. The points of different colors belong to different samples (groups). Each point represents one sample. The closer the distance between two points, the higher the similarity, and the smaller the difference in the microbial community structure between the two samples. (e) Partial least squares discriminant analysis (PLS-DA); each point represents a sample, points of the same color belong to the same group, and points of the same group are marked with ellipses. If the samples belonging to the same grouping are closer to each other and the distance between the points of different grouping is farther, the classification model is better.

\section{Discussion}

The results in this study demonstrated that dietary supplementation with both TCM prescriptions could reduce diarrhea rate, promote growth performance, suppress TLR4/ MyD88/NF- $\kappa \mathrm{B}$ signaling pathways, and decrease the levels of proinflammatory cytokine expression in the colon of piglets. Accordingly, the 16S rRNA analysis revealed that the component and relative abundances of beneficial bacteria were altered in both TCM groups, compared to the control.
During the nursing period, piglets are prone to colonic dysfunction, leading to diarrhea and edema, which results in impaired growth performance or even death [21, 22]. Previous study reported that supplementation of medicinal plants or its extracts increases growth performance in pigs, mainly because the active ingredient could have antimicrobial properties, aid in the decrease in intestinal $\mathrm{pH}$ and against diarrhea, and improve nutrient digestibility [22, 23]. Meanwhile, reducing the $\mathrm{pH}$ value of gut contents could inhibit or eliminate some pathogenic flora to prevent 


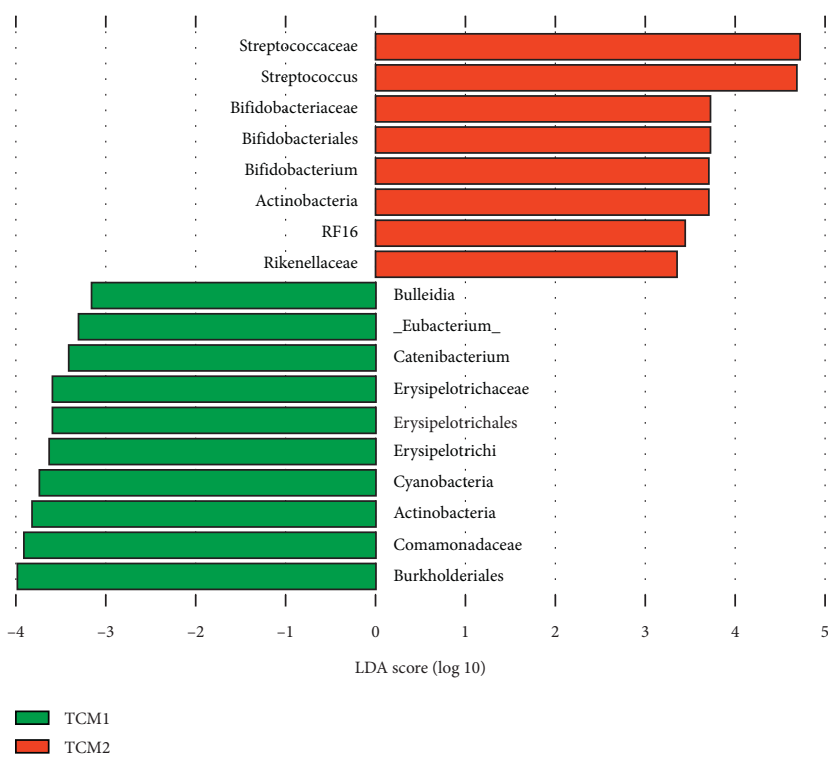

(a)

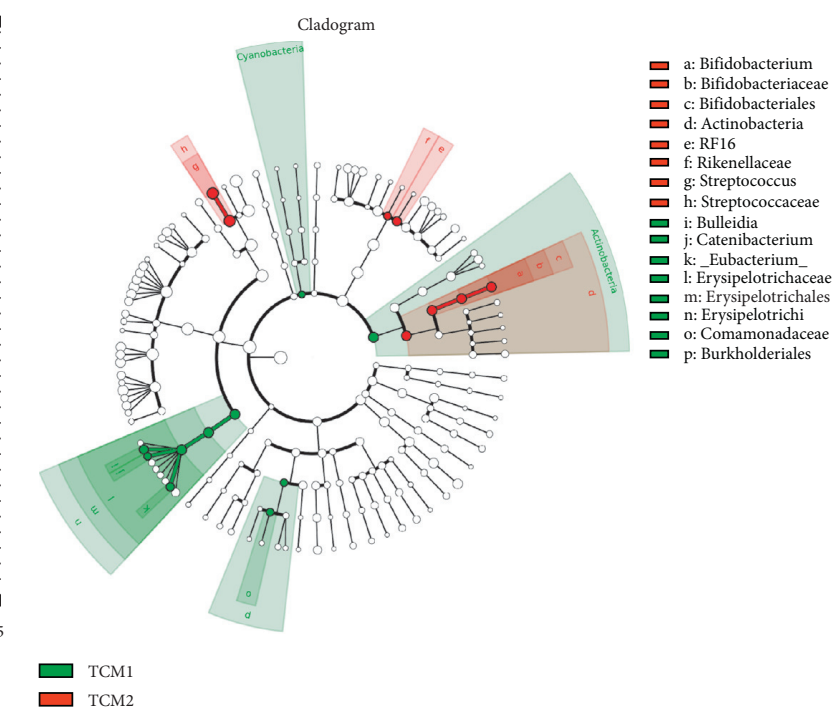

(b)

FIGURE 6: Linear discriminant analysis effect size (LEFse) analysis on caecum microbiota. (a) Linear discriminant analysis (LDA): an LDA score higher than 2 indicated a higher relative abundance in the corresponding group than in the other two groups. (b) LEfSe taxonomy cladogram: different colors suggest enrichment of certain taxa in TCM1 (blue) and TCM2 (red). The size of the circles is based on relative abundance. The significantly different species using the nonparametric factorial Kruskal-Wallis rank sum test at a significance level of 0.05 .

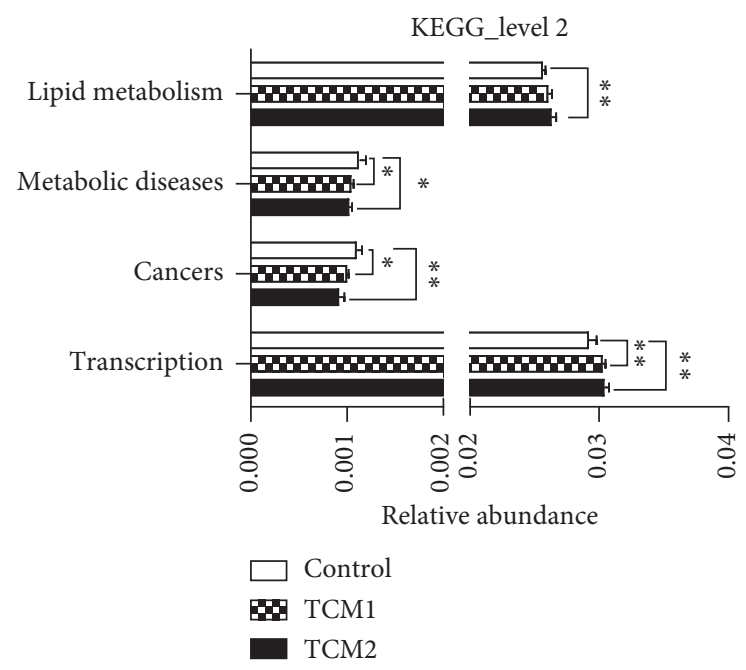

FIGURE 7: Microbiome function prediction according to the KEGG pathway database. The horizontal coordinates represent the relative abundance of functional genes in different groups; the vertical coordinates represent the functions of genes. The "*” or “**” indicates a significant difference $(P<0.05)$ or a highly significant difference $(P<0.01)$ between treatment groups, respectively.

diarrhea via improving gut health status in piglets. In this study, both TCM prescriptions significantly decreased the diarrhea rate and colon or caecum $\mathrm{pH}$ value in piglets, which suggested that TCM prescriptions were effective for diarrhea control, and it may attribute to the TCM capable of improving the intestinal environment directly or indirectly.

Weaning or other stresses can damage the growth performance of piglets, via decreasing the ADG and ADFI.
In this study, the difference was not significant in the ADFI among all groups, which may be because the TCM additives slightly changed the taste of the feed. Notably, our results indicated that both TCM1 and TCM2 promoted ADG and decreased the F: G ratio remarkably, indicating that both treatments promoted growth performance in piglets. The improvement of growth performance is associated with better intestinal environment (intestinal $\mathrm{pH}$ ) and the promotion of energy intake by elevating apparent nutrient digestibility. Meanwhile, intestinal peristalsis improvement and acidic environment contribute to increasing the digestion and absorption of nutrients and improving the nutritional digestibility of piglets, subsequently promoting growth [24], which is of great significance for piglets with the imperfect digestive system. Huang et al. suggested that TCM supplementation increased the apparent nutrient digestibility such as dietary dry matter, CP, and gross energy in pigs, which is consistent with our results [24]. Moreover, our data showed that the digestibility of $\mathrm{CP}, \mathrm{CF}, \mathrm{NDF}, \mathrm{ADF}, \mathrm{Ca}$, and $\mathrm{P}$ was increased in TCM1 treatment, but supplementation with TCM2 has no significant effect on CF, Ca, and P absorption, indicating that TCM1 exerts a more comprehensive effect on apparent digestibility than that of TCM2. Additionally, the changes in various nutrient digestibility may be also associated with the alleviation in intestinal inflammation and improvement of gut microbiota, much likely due to TCM1 and TCM2.

It is well established that different regions of the gut have distinct physiological functions. Large intestine has optimal conditions for prolific bacterial growth: warm, moist, anaerobic, and filled with feed residues that flow at a relatively low speed. Many kinds of pathogens, such as spirochetes, pass through the small intestine until ultimately colonizing 


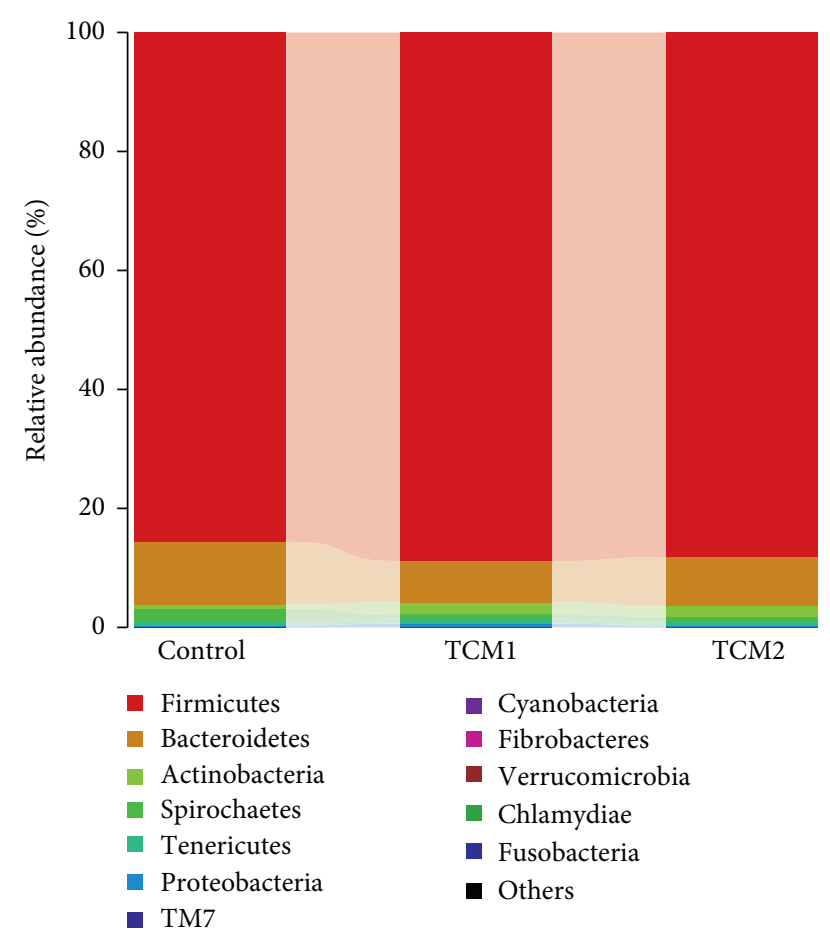

FIgURE 8: Bacterial composition of the different groups at the phylum level.

and proliferating in the colon, which increases the risk of bacterial infection and inflammatory response $[25,26]$. Meanwhile, previous study has demonstrated that TLR4 and CD14 are expressed higher in the colon than intestine [27]. Thus, given the highest correlation between colon immunity with TLR4/MyD88/NF- $\kappa$ B signaling pathway, we examined the expression of inflammation-related genes and proteins in the TLR4/MyD88/NF- $\kappa$ B signaling pathway, as well as the inflammatory cytokines downstream.

Although there are various causes of diarrhea, intestinal inflammation is usually involved. The TLR4/MyD88/NF- $\kappa \mathrm{B}$ signaling pathway is one of the major pathways mediating inflammatory responses in the intestine, which is triggered by pattern recognition receptors (PRRs) and TLRs via interacting with the antigen from gut microbiota [28]. TLR4 is essential for LPS-mediated signaling. After binding to TLR4, LPS activates the MyD88-dependent pathway, a key inflammatory signaling pathway [29]. The activation of MyD88 will phosphorylate $\mathrm{I} \kappa \mathrm{B}$ kinase, which in turn activates the transcription factor $\mathrm{NF}-\kappa \mathrm{B}$ and triggers the downstream expression of proinflammatory cytokines along with other immune-related genes [30]. NF- $\kappa$ B resides outside the nucleus in its inactive form bound to inhibitory $\mathrm{I} \kappa \mathrm{B}$ $\alpha$. Upon activation, the protein levels of NF- $\kappa \mathrm{B}$ p65, p-NF$\kappa \mathrm{B}$ p65, and $\mathrm{p}-\mathrm{I} \kappa \mathrm{B}-\alpha$ were increased and the levels of $\mathrm{I} \kappa \mathrm{B}-\alpha$ were decreased [31]. In this study, both TCM treatments downregulated NF- $\kappa \mathrm{B}$ p $65, \mathrm{p}-\mathrm{NF}-\kappa \mathrm{B}$ p 65 , and $\mathrm{p}-\mathrm{I} \kappa \mathrm{B}-\alpha$ protein levels, upregulated the $\mathrm{I} \kappa \mathrm{B}-\alpha$ protein levels, and significantly reduced the ratio of phosphorylated protein $(\mathrm{p}-\mathrm{NF}-\kappa \mathrm{B}$ p65 and $\mathrm{p}-\mathrm{I} \kappa \mathrm{B}-\alpha)$ to total protein, which is consistent with $\mathrm{Xu}$ et al. [32], thus endorsing the anti- inflammatory activity of TCM1 and TCM2. Importantly, in TCM groups, the results of inflammation-related genes in the TLR4/MyD88/NF- $\kappa \mathrm{B}$ signaling pathway and NF- $\kappa \mathrm{B}$ p65, p-NF- $\kappa \mathrm{B}$ p $65, \mathrm{I} \kappa \mathrm{B}-\alpha$, and $\mathrm{p}-\mathrm{I} \kappa \mathrm{B}-\alpha$ protein levels were in agreement with the lower levels of inflammatory cytokine, which indicated that TCM relieved inflammation by suppressing the activity of TLR4/MyD88/NF- $\kappa \mathrm{B}$ signaling pathway in the colon. Moreover, these changes may be associated with the TCM-induced amelioration of gut microbiota diversity or abundance [33].

The interaction between microbiota and the host influences immunological homeostasis, and the changes in this interaction are associated with various inflammatory cytokines. Previous study has proved that TCM prescription decreased inflammatory cytokine level that was related to the increased abundance of beneficial gut microbiota and decreased LPS levels [34]. Furthermore, certain components in Chinese herbal medicines exert their pharmacological effects through interactions with gut bacteria $[14,35]$. Importantly, disturbed gut microbiota, in either diversity or abundance, have been found to play an important role in the pathological development of inflammatory bowel disease (IBD) [33]. In present study, TCM groups significantly elevated the richness or diversity of caecum microbiota. Also, according to the NMDS and PLS-DA analysis, we observed significant structural changes in the caecum microbiota in TCM1 and TCM2 groups compared to the control. All of these results indicated that experimental groups TCM1 and TCM2 exhibited different caecum microbiota structures compared to the control group.

Our results showed that the main phyla were Firmicutes, followed by Bacteroidetes in caecum bacterial communities of piglets (Figure 8), which is in accordance with previous study [35]. The study indicated that supplementation of TCM or its extracts can ameliorate the structure of gut microbiota by increasing probiotics and reducing pathogens, leading to decreased risk of IBD $[36,37]$. In current study, TCM1 significantly increased the caecum phylum Cyanobacteria and Actinobacteria. As reported, Cyanobacteria play a vital role in inhibiting inflammation by production of antiinflammatory pitinoic acids B and C [38]. Meanwhile, at the genus level, TCM1 increased the abundance of Eubacterium, Bulleidia, and Catenibacterium, respectively, and that of Streptococcus and Bifidobacterium were elevated in the TCM2 group. Gut probiotics may be effective for secondary prevention in patients with recurrent Clostridium difficileassociated diarrhea through maintenance of the normal gastrointestinal flora [39]. As reported, Bulleidia could ferment glucose and anti-inflammatory products acetate, lactate, and trace amounts of succinate were the end products of fermentation [40]. A few studies demonstrated that Eubacterium and Bifidobacteria were able to use carbohydrate for the production of short-chain fatty acids, which can inhibit inflammatory responses in the gut [41, 42]. Bifidobacteria are also demonstrated to modulate the intestinal immune system and have been shown to increase the production of immunoglobulin A [42]. Apparently, an increase in the abundance of these beneficial bacteria species may be related to the alleviation in intestinal inflammation 
by TCM treatments. Conversely, intestinal bacteria can also alleviate inflammation by inducing improvements to intestinal epithelial barrier integrity [43]. Furthermore, this also may be associated with the AAs, a rich component of TCM, such as aspartic acid and glutamic acid, could be available to the microbiota in the gut in mammals, and play a critical role in regulating the intestinal mucosal immunity and microbiota directly or indirectly, contributing to intestinal homeostasis $[14,44]$.

Indeed, gut microbiota not only experiences long-term coevolution with host [45] but also regulates the host immunity [46]. Especially, lifestyle, diets, and medicine intake contributes greatly to the alteration of gut microbiota [47]. In current research, it was found that TCM1 and TCM 2 are mainly composed of antibacterial and antitumor active ingredients such as volatile oil, alkaloids, and flavone. Thus, we explored microbiota function based on inferred metagenomes using the PICRUSt algorithm [48]. Our analysis of inferred metagenomes revealed decreased abundance of human disease pathways, including the metabolic diseases and cancers in TCM treatments compared to the control. This finding suggests that, aside from decreasing intestinal $\mathrm{pH}$ and inhibiting inflammation, increasing abundance of beneficial bacteria species after TCM intervention is also known to have prominent effects on metabolic diseases and cancers.

\section{Conclusion}

Dietary supplementation with TCM1 and TCM2 effectively reduced the diarrhea rate in piglets and promoted growth performance by improving the gut physical circumstance, nutrient digestibility, colonic inflammation alleviation, and caecum microbial composition amelioration. Importantly, TCM prescription may be a potential therapeutic strategy for piglet diarrhea.

\section{Data Availability}

The data used to support the findings of this study are available from the corresponding author upon request.

\section{Disclosure}

Jian Chen and Yaqing Mao are the co-first authors.

\section{Conflicts of Interest}

The authors declare that they have no conflicts of interest.

\section{Acknowledgments}

All authors thank all members of the team for their help in the experimental process in clinical veterinary medicine laboratory in the College of Animal Science and Technology, Jiangxi Agricultural University. This work was supported by Major Research and Development Projects of Jiangxi Province (20194ABC28008) and Spirit Jinyu Biological Pharmaceutical Co., Ltd. (Huhhot, Inner Mongolia, China).

\section{References}

[1] H. Yi, L. Zhang, Z. Gan et al., "High therapeutic efficacy of cathelicidin-WA against postweaning diarrhea via inhibiting inflammation and enhancing epithelial barrier in the intestine," Scientific Reports, vol. 6, no. 1, Article ID 25679, 2016.

[2] P. Bin, Z. Tang, S. Liu et al., "Intestinal microbiota mediates enterotoxigenic Escherichia coli-induced diarrhea in piglets," BMC Veterinary Research, vol. 14, no. 1, 2018.

[3] L. Jean-Paul, B. Paolo, S. Hauke, and C. R. Stokes, "Nutritional management of gut health in pigs around weaning," Proceedings of the Nutrition Society, vol. 66, no. 2, pp. 260-268, 2007.

[4] S. Monroe and R. Polk, "Antimicrobial use and bacterial resistance," Current Opinion in Microbiology, vol. 3, no. 5, pp. 496-501, 2000.

[5] G. P. P. Kamatou, N. P. Makunga, W. P. N. Ramogola, and A. M. Viljoen, "South african salvia species: a review of biological activities and phytochemistry," Journal of Ethnopharmacology, vol. 119, no. 3, pp. 664-672, 2008.

[6] W. Lan, Z. Guang-Biao, L. Ping et al., "Dissection of mechanisms of Chinese medicinal formula realgar-Indigo naturalis as an effective treatment for promyelocytic leukemia," Proceedings of the National Academy of Sciences of the United States of America, vol. 105, no. 12, pp. 4826-4831, 2008.

[7] Anonymous, Formularies for 52 Kinds of Disorders, Culture Relics Press, Beijing, China, 1979.

[8] Anonymous, The Inner Canon of Emperor Huang, Chinese Medical Ancient Books Publishing House, Beijing, China, 2003.

[9] C. T. Keith, A. A. Borisy, and B. R. Stockwell, "Multicomponent therapeutics for networked systems," Nature Reviews Drug Discovery, vol. 4, no. 1, pp. 71-78, 2005.

[10] P. P. Tak and G. S. Firestein, "NF- $\kappa$ B: a key role in inflammatory diseases," Journal of Clinical Investigation, vol. 107, no. 1, pp. 7-11, 2001.

[11] H. Liu, P. Lv, H. Wu et al., "Structural modulation of gut microbiota during alleviation of suckling piglets diarrhoea with herbal formula," Evidence-Based Complementary and Alternative Medicine, vol. 2017, Article ID 8358151, 11 pages, 2017.

[12] V. Gopalakrishnan, C. N. Spencer, L. Nezi et al., "Gut microbiome modulates response to anti-PD-1 immunotherapy in melanoma patients," Science, vol. 359, no. 6371, pp. 97-103, 2018.

[13] H. J. Haiser, D. B. Gootenberg, C. Kelly, S. Gopal, E. P. Balskus, and P. J. Turnbaugh, "Predicting and manipulating cardiac drug inactivation by the human gut bacterium Eggerthella lenta," Science, vol. 341, no. 2, pp. 295-298, 2014.

[14] N. Ma and X. Ma, "Dietary amino acids and the gutmicrobiome-immune axis: physiological metabolism and therapeutic prospects," Comprehensive Reviews in Food Science and Food Safety, vol. 18, no. 1, pp. 221-242, 2019.

[15] Q. Shu, F. Qu, and H. S. Gill, "Probiotic treatment using Bifidobacterium lactis HN019 reduces weanling diarrhea associated with rotavirus and Escherichia coli infection in a piglet model," Journal of Pediatric Gastroenterology and Nutrition, vol. 33, no. 2, pp. 171-177, 2001.

[16] K. Guo, H. Cao, Y. Zhu et al., "Improving effects of dietary rumen protected $\gamma$-aminobutyric acid additive on apparent nutrient digestibility, growth performance and health status in heat-stressed beef cattle," Animal Science Journal, vol. 89, no. 9, pp. 1280-1286, 2018. 
[17] D. N. Miller, J. E. Bryant, E. L. Madsen, and W. C. Ghiorse, "Evaluation and optimization of DNA extraction and purification procedures for soil and sediment samples," Applied \& Environmental Microbiology, vol. 65, no. 11, pp. 4715-4724, 1999.

[18] L. Zhao, G. Wang, P. Siegel et al., "Quantitative genetic background of the host influences gut microbiomes in chickens," Scientific Reports, vol. 3, no. 5, p. 1163, 2013.

[19] J. G. Caporaso, J. Kuczynski, J. Stombaugh et al., "QIIME allows analysis of high-throughput community sequencing data," Nature Methods, vol. 7, no. 5, pp. 335-336, 2010.

[20] S. Jiyan, S. Lu, L. Dan et al., "Antitumor activity of extract from the sporoderm-breaking spore of ganoderma lucidum: restoration on exhausted cytotoxic T cell with gut microbiota remodeling," Frontiers in Immunology, vol. 9, p. 1765, 2018.

[21] K. Jung, B.-K. Kang, C.-S. Lee, and D.-S. Song, "Impact of porcine group a rotavirus co-infection on porcine epidemic diarrhea virus pathogenicity in piglets," Research in Veterinary Science, vol. 84, no. 3, pp. 502-506, 2008.

[22] X. F. Kong, G. Y. Wu, Y. P. Liao et al., "Effects of Chinese herbal ultra-fine powder as a dietary additive on growth performance, serum metabolites and intestinal health in earlyweaned piglets," Livestock Science, vol. 108, no. 1-3, pp. 272-275, 2007.

[23] S. K. Kommera, R. D. Mateo, F. J. Neher, and S. W. Kim, "Phytobiotics and organic acids as potential alternatives to the use of antibiotics in nursery pig diets," Asian-australasian Journal of Animal Sciences, vol. 19, no. 12, pp. 1784-1789, 2006.

[24] C. W. Huang, T. T. Lee, Y. C. Shih, and B. Yu, "Effects of dietary supplementation of Chinese medicinal herbs on polymorphonuclear neutrophil immune activity and small intestinal morphology in weanling pigs," Journal of Animal Physiology and Animal Nutrition, vol. 96, no. 2, pp. 285-294, 2012.

[25] A. M. Mowat and W. W. Agace, "Regional specialization within the intestinal immune system," Nature Reviews Immunology, vol. 14, no. 10, pp. 667-685, 2014.

[26] E. R. Burrough, "Swine dysentery: etiopathogenesis and diagnosis of a reemerging disease," Veterinary Pathology, vol. 54, no. 1, pp. 22-31, 2016.

[27] W. Yunwei, D. Suzanne, W. Mark et al., "Regional mucosaassociated microbiota determine physiological expression of tlr2 and tlr4 in murine colon," PLoS One, vol. 5, no. 10, Article ID e13607, 2010.

[28] Y. Ma, M. He, and L. Qiang, "Exercise therapy downregulates the overexpression of TLR4, TLR2, MyD88 and NF- $\kappa \mathrm{B}$ after cerebral ischemia in rats," International Journal of Molecular Sciences, vol. 14, no. 2, pp. 3718-3733, 2013.

[29] Y.-C. Lu, W.-C. Yeh, and P. S. Ohashi, "LPS/TLR4 signal transduction pathway," Cytokine, vol. 42, no. 2, pp. 145-151, 2008.

[30] S. Akira, K. Takeda, and T. Kaisho, "Toll-like receptors: critical proteins linking innate and acquired immunity," Nature Immunology, vol. 2, no. 8, pp. 675-680, 2001.

[31] S. Mitchell, J. Vargas, and A. Hoffmann, "Signaling via the $\mathrm{NF} \kappa \mathrm{B}$ system," Wiley Interdisciplinary Reviews: Systems Biology and Medicine, vol. 8, no. 3, pp. 227-241, 2016.

[32] X. Xu, L. Zhang, Z. Liu et al., "Therapeutic efficacy of the traditional Chinese medicine baishaoqiwu on TNBS-induced colitis is associated with down-regulation of the TLR4/ MyD88/NF- $\kappa$ B Signaling Pathway," Vivo, vol. 30, no. 3, pp. 181-186, 2016.

[33] R. Ignacio, G. M. Peter, W. Roger, S. Fei, and H. H. Elisabeth, "Degree of colitis correlates with microbial composition and cytokine responses in colon and caecum of Gai2-deficient mice," Fems Microbiology Ecology, vol. 92, no. 7, p. fiw98, 2016.

[34] Y. Zhang, K. Tang, Y. Deng et al., "Effects of shenling baizhu powder herbal formula on intestinal microbiota in high-fat diet-induced nafld rats," Biomedicine \& Pharmacotherapy, vol. 102, pp. 1025-1036, 2018.

[35] P. Eun-Kyung, S. Jieun, B. Eun-Ah, L. Young-Chul, and K. Dong-Hyun, "Intestinal bacteria activate estrogenic effect of main constituents puerarin and daidzin of Pueraria thunbergiana," Biological \& Pharmaceutical Bulletin, vol. 29, no. 12 , pp. 2432-2435, 2006.

[36] Q. Niu, P. Li, S. Hao et al., "Dynamic distribution of the gut microbiota and the relationship with apparent crude fiber digestibility and growth stages in pigs," Scientific Reports, vol. 5, no. 1, p. 9938, 2015.

[37] C. J. Chang, C. S. Lin, C. C. Lu et al., "Corrigendum: ganoderma lucidum reduces obesity in mice by modulating the composition of the gut microbiota," Nature Communications, vol. 6, p. 7489, 2017.

[38] Z. Xu, Z. Yufeng, Z. Menghui et al., "Structural changes of gut microbiota during berberine-mediated prevention of obesity and insulin resistance in high-fat diet-fed rats," PLoS One, vol. 7, no. 8, Article ID e42529, 2012.

[39] M. Rana, V. J. Paul, and L. Hendrik, "Modular strategies for structure and function employed by marine cyanobacteria: characterization and synthesis of pitinoic acids," Organic Letters, vol. 15, no. 16, pp. 4050-4053, 2013.

[40] J. R. Crow, S. L. Davis, D. M. Chaykosky, T. T. Smith, and J. M. Smith, "Probiotics and fecal microbiota transplant for primary and secondary prevention of clostridium difficile infection," Pharmacotherapy, vol. 35, no. 11, pp. 1016-1025, 2016.

[41] J. Downes, B. Olsvik, S. J. Hiom et al., "Bulleidia extructa gen. nov., sp. nov., isolated from the oral cavity," International Journal of Systematic and Evolutionary Microbiology, vol. 50, no. 3, p. $979,2000$.

[42] C. Belzer, L. W. Chia, S. Aalvink et al., "Microbial metabolic networks at the mucus layer lead to diet-independent butyrate and vitamin B 12 production by intestinal symbionts," mBio, vol. 8, no. 5, pp. e717-e770, 2017.

[43] D. D. Heeney, Z. Zhai, Z. Bendiks et al., "Lactobacillus plantarum bacteriocin is associated with intestinal and systemic improvements in diet-induced obese mice and maintains epithelial barrier integrity in vitro," Gut Microbes, vol. 10, no. 3, pp. 382-397, 2018.

[44] K. C. Nagulapalli Venkata, A. Swaroop, D. Bagchi, and A. Bishayee, "A small plant with big benefits: fenugreek (Trigonella foenum-graecum linn.) for disease prevention and health promotion," Molecular Nutrition \& Food Research, vol. 61, no. 6, Article ID 1600950, 2017.

[45] A. H. Moeller, Y. Li, E. Mpoudi Ngole et al., "Rapid changes in the gut microbiome during human evolution," Proceedings of the National Academy of Sciences, vol. 111, no. 46, pp. 16431-16435, 2014.

[46] A. Ouwehand, E. Isolauri, and S. Salminen, "The role of the intestinal microflora for the development of the immune system in early childhood," European Journal of Nutrition, vol. 41, pp. i32-i37, 2002.

[47] L. Yang, S. Liu, J. Ding et al., "Gut microbiota co-microevolution with selection for host humoral immunity," Frontiers in Microbiology, vol. 8, p. 1243, 2017.

[48] M. G. I. Langille, J. Zaneveld, J. G. Caporaso et al., "Predictive functional profiling of microbial communities using $16 \mathrm{~S}$ rRNA marker gene sequences," Nature Biotechnology, vol. 31, no. 9, pp. 814-821, 2013. 